\title{
Automatic Detection of Characteristic Waves in Electrocardiogram
}

\author{
Lucia Billeci ${ }^{1}$, Lorenzo Bachi ${ }^{1}$, Maurizio Varanini ${ }^{1}$ \\ ${ }^{1}$ Institute of Clinical Physiology, National Research Council of Italy (IFC-CNR), Pisa, Italy
}

\begin{abstract}
The goal of automatic ECG analysis is to assess the clinical status of the heart system as accurately as possible, and the identification of $P$ and $T$ waves plays a significant role in this matter.

This works presents original algorithms for the detection of $P$ and $T$ waves. These algorithms are based on the morphological and temporal characteristics of the electrocardiogram. To test and compare the algorithms' performance, we considered the QTDB and MIT-BIH Arrhythmia annotated databases.

The developed algorithms obtained a good performance for the detection of both peaks. In particular, in both the QTDB and MIT-BITH database the $P$ wave detection algorithm obtained considerably higher performance than those presented in the literature (QTDB: $95.87 \%$ vs 89.05\%; MIT-BITH: $84.65 \%$ vs $83.36 \%$ for Lead 1). The T wave detection algorithm, achieved best performance than those in literature in the QTDB (89.05\% vs 87.49\%) while in the MIT-BITH database results were almost comparable to those reported in the literature. These findings suggest the high potential of the proposed simple algorithms for $P$ and $T$ wave detection in ECG.
\end{abstract}

\section{Introduction}

A typical ECG consists of a quasi-periodic succession of groups of waves (PQRST) representing the cardiac cycle. The $\mathrm{P}$ wave represents the depolarization that spreads from the sino-atrial node throughout the atria, the QRS complex corresponds to the ventricular depolarization, while the $\mathrm{T}$ wave corresponds to the ventricular repolarization phase of the heart cycle.

The morphology of the $\mathrm{P}$ wave provides relevant information concerning intra-atrial conduction, hypertrophic conditions of the atria and atrioventricular conduction. In some pathological conditions the morphology of the $\mathrm{T}$ wave may change from beat to beat [1]. The correct identification of $\mathrm{P}$ and $\mathrm{T}$ waves is extremely important for an appropriate diagnosis of cardiac problems. In particular, accurate $\mathrm{P}$ wave detection and recognition of its variations is relevant in clinical diagnosis of supra-ventricular arrhythmia as well as for confirming the presence of ventricular arrhythmia [2]. Moreover, the exact delineation of $\mathrm{P}$ wave is required in the identification of atrial fibrillation [3, 4]. On the other side, the detection and delineation of $\mathrm{T}$ wave is required for the identification of potentially fatal arrhythmia, myocardial infarction and acute coronary syndrome [5].

The identification of $\mathrm{P}$ and $\mathrm{T}$ waves is traditionally performed by cardiologists which visually inspect signal morphology. This process is time-consuming and requires expert human resources with specialized education and practice. Therefore, the automatic analysis of ECG for the detection of characteristic waves can be a useful tool for the early detection of cardiac abnormalities and the prevention of their quick progress.

Although different automatic approaches have been proposed in literature for the detection of on, off and peak location of $\mathrm{P}$ and $\mathrm{T}$ wave of the ECG signal [6,7], the results are still unsatisfactory. This is also due to the limited availability of annotated databases on which the algorithms can be trained and tested. Moreover, these methods have often a high computational cost, making them unusable for real-time applications. Furthermore, the performances of these methods are often not comparable because the Authors use different tolerance window in defining True Positive (TP) events.

In this paper, we present a novel algorithm which is based on the morphological and temporal characteristics of $\mathrm{ECG}$, for a fast and accurate detection of $\mathrm{P}$ and $\mathrm{T}$ waves.

\section{Methods}

Two different algorithms were implemented for $\mathrm{P}$ wave and $\mathrm{T}$ wave detection. These algorithms were applied on the two leads of the selected databases. Since the ability to detect $\mathrm{P}$ and $\mathrm{T}$ waves critically depends on the correct positioning of $\mathrm{R}$ peaks, our algorithms were evaluated considering annotated $R$ peaks. The performance of the $P$ wave and $T$ wave detection algorithms was then determined by comparing the detected fiducial points with the annotation of the $\mathrm{P}$ and $\mathrm{T}$ wave in the databases. Specifically, the peaks were detected for comparison with 
the performance of the algorithm in [7].

\subsection{Data}

The algorithms developed in this study were tested on two different databases freely available from the PhysioNet portal: the MIT-BIH Arrhythmia Database [8] and the QT database [9]. Both the two databases are annotated for QRS complexes and $\mathrm{P}$ and $\mathrm{T}$ waves annotations. Table 1 summarize the characteristics of the two databases. The algorithms were tested on both available leads for each record.

The MIT-BIH arrhythmia database consists of 48 30min ECG registrations obtained with a sampling frequency of $360 \mathrm{~Hz}$. For consistency with the study by Friganovic et al. [7] the following records were excluded from the analysis: 102, 104, 107, 207 and 217. This database includes $\mathrm{R}$ peaks annotation while $\mathrm{P}$ and $\mathrm{T}$ waves annotations are provided in [5].

The QT database includes 105 15-minutes ECG records, sampled at (or resampled to) $250 \mathrm{~Hz}$. Only the last 5 minutes of the records are annotated. The records in QT Database were taken from seven different databases. Between 30 and 100 representative beats were manually annotated by cardiologists in each record, who identified the onset, peak, and offset of P waves, onset, peak, and offset of QRS complexes, the peak and offset of T waves, and (if present) the peak and offset of U-waves [9]. Annotations in .atr format, reference beat annotations from the original database, were recalculated for record sel232 in the QT database on the basis of the record 232 annotations from the MIT-BIH Arrhythmia Database.

Table 1. Characteristics of the two databases used in this study.

\begin{tabular}{lllll}
\hline Database & $\begin{array}{l}\text { No. of } \\
\text { records }\end{array}$ & $\begin{array}{l}\text { No. of } \\
\text { leads }\end{array}$ & P peaks & T peaks \\
\hline $\begin{array}{l}\text { MIT-BIH } \\
\text { arrhythmia } \\
\text { database }\end{array}$ & 43 & 2 & 95989 & 98395 \\
QT database & 105 & 2 & 3194 & 3542 \\
\hline
\end{tabular}

\subsection{Pre-processing}

The first pre-processing step was aimed at eliminating power-line interference which consists in a sinusoidal component at a frequency around $50 \mathrm{~Hz}(60 \mathrm{~Hz}$ in the US) and its harmonics. The power spectral density was estimated by the Welch method (averaged windowed periodogram, eight sections with 50\% overlap, Hamming window). The existence of a power-line component was assessed by comparing the peak of the power density in a narrow interval around $50 \mathrm{~Hz}$ and around $60 \mathrm{~Hz}$ with the average power density in the neighbors of such frequencies. If power-line interference was detected, it was removed by applying notch filters (forward-backward, zero phase, $1 \mathrm{~Hz}$ bandwidth) at the detected peak frequency and its next three harmonics.

Baseline wonder removal was realized by calculating a line passing through the $\mathrm{Q}$ wave onset (Qon) points. More precisely, the average values in an interval of $10 \mathrm{~ms}$ centered on the Qon points was used. For the search of Qon points an algorithm based on derivatives and amplitude of the signal was applied [10]. The detrended signal was obtained as the difference between the original and baseline signal.

Movement and artifact canceling was obtained by the application of a low pass filtering realized with a moving average on $0.35 \mathrm{~s}$. Finally, a $1000 \mathrm{~Hz}$ signal interpolation was applied to better localize fiducial points.

\subsection{P wave detection}

The P-wave peak (Ppeak) detection algorithm was aimed at maximize the area subtended between the signal and a line hinged to a signal in two points separated by a temporal distance of $0.08 \mathrm{~s}$. Specifically, this line started with its right extremity in correspondence of the Qon point and was drawn backward until its left extremity reached an estimate $\mathrm{T}$ wave end position obtained using the previous beat QRS annotation and applying the Bazett's formula [11]

\subsection{T wave detection}

The algorithm for T-wave peak (Tpeak) detection is based on the identification of an interval of interest as the last $2 / 3$ of the segment from QRS end to the T-wave position as identified by the Bazett's formula [11]. Some interval adjustments were applied. Indeed, T-wave can occur, on a specific lead, with different shape. Commonly $\mathrm{T}$ wave is positive and its peak is well defined, however it may be also negative or biphasic (Figure 1). In order to manage this polymorphism of the T-wave, our algorithm started searching, in the selected interval, for the maximum of absolute value of the signal. After that, it compared the curvature of the original signal around such maximum with the curvature around the true maximum of the original signal.

\section{Results}

The performance of the proposed algorithms was evaluated in terms F1 score (F1). This measure was calculated as follows:

$$
F 1=\frac{2 T P}{2 T P+F P+F N}
$$




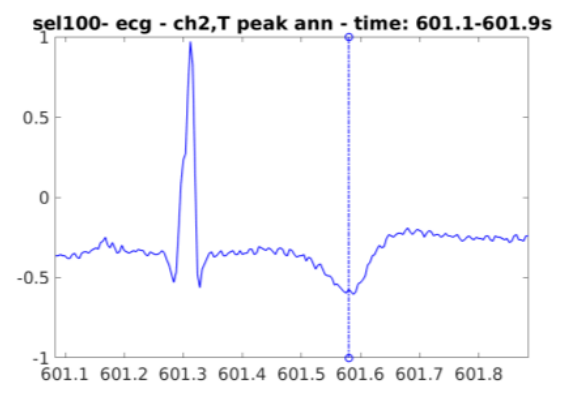

a

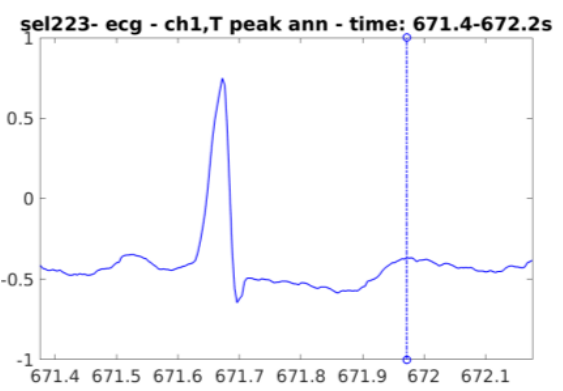

b

Figure 1: Examples of $\mathrm{T}$ waves shapes. a) Negative peak, b) Biphasic.

For $\mathrm{P}$ and $\mathrm{T}$ waves peak detection, the detected peak was considered a true positive (TP) if its position was in the range of $\pm 75 \mathrm{~ms}$ from the annotated peak, otherwise, it was considered a false positive (FP) [12].

Table 2 summarizes the performance of our algorithm in the identification of $\mathrm{P}$ and $\mathrm{T}$ wave peaks in terms of $\mathrm{F} 1$ for both leads for every database. Our results were compared with the algorithm obtaining the best performance as indicated in [7]. For each channel and database, the best results are emphasized in bold.

Table 2. Performance of the proposed algorithm for the identification of $\mathrm{P}$ and $\mathrm{T}$ peak.

\begin{tabular}{lllll}
\hline Lead & \multicolumn{2}{c}{ F1 Ppeak (\%) } & \multicolumn{2}{c}{ F1 Tpeak (\%) } \\
\hline & $\begin{array}{l}\text { This } \\
\text { work }\end{array}$ & Best in [7] & This & Best in [7] \\
& \multicolumn{3}{c}{ work } \\
\hline QTDB ch1 & $\mathbf{9 5 . 8 7}$ & 89.64 & $\mathbf{8 9 . 0 5}$ & 87.49 \\
QTDB ch2 & $\mathbf{9 2 . 5 2}$ & 86.92 & $\mathbf{8 8 . 7 0}$ & 88.50 \\
& & & & \\
MIT-BIH ch1 & $\mathbf{8 4 . 6 5}$ & 83.37 & 88.39 & $\mathbf{9 1 . 8 3}$ \\
MIT-BIH ch2 & $\mathbf{7 8 . 3 2}$ & 78.09 & $\mathbf{6 4 . 5 0}$ & 60.68 \\
\hline
\end{tabular}

It is important to notice that not the same algorithm obtained the best performance in [7] nor for the two databases neither for the two waves or even for the two leads. In Table 3 we reported the performance of our algorithm for Ppeak detection compared with the performances of the algorithms which obtained at least one of the best performances in [7].
Table 3. Summary performance for P peak detection.

\begin{tabular}{lccc}
\hline Method & QTDB & MIT-BIH & Mean \\
\hline \multicolumn{4}{c}{ Lead 1 } \\
This work & $\mathbf{9 5 . 8 7}$ & $\mathbf{8 4 . 6 5}$ & $\mathbf{9 0 . 2 6}$ \\
Martinez WT & 85.17 & 76.69 & 80.93 \\
Martinez WT + PT & 89.64 & 83.37 & 86.50 \\
Martinez WT + & 86.78 & - & - \\
templates & & & \\
MMF + Elgendi & 46.27 & 54.39 & 50.33 \\
\hline & Lead 2 & & \\
\hline This work & $\mathbf{9 2 . 5 2}$ & $\mathbf{7 8 . 3 2}$ & $\mathbf{8 5 . 4 2}$ \\
Martinez WT & 85.82 & 67.58 & 76.70 \\
Martinez WT + PT & 86.92 & 78.09 & 82.50 \\
Martinez WT + & 86.75 & - & - \\
templates & & & 43.41 \\
MMF + Elgendi & 42.07 & 44.75 & \\
\hline
\end{tabular}

In Table 4 we reported the performance of our algorithm for Tpeak detection compared with the performances of the algorithms which obtained at least one of the best performances in [7].

Table 4. Summary performance for T peak detection.

\begin{tabular}{|c|c|c|c|}
\hline Method & QTDB & MIT-BIH & Mean \\
\hline \multicolumn{4}{|c|}{ Lead 1} \\
\hline This work & 89.05 & 88.39 & 88.72 \\
\hline Martinez WT & 86.91 & 68.23 & 77.57 \\
\hline Martinez WT + PT & 84.99 & 71.53 & 78.26 \\
\hline $\begin{array}{l}\text { Martinez WT + } \\
\text { templates }\end{array}$ & 87.49 & - & - \\
\hline MMF + Elgendi & 76.13 & 91.83 & 83.98 \\
\hline \multicolumn{4}{|c|}{ Lead 2} \\
\hline This work & 88.70 & 64.60 & 76.65 \\
\hline Martinez WT & 88.46 & 60.68 & 74.57 \\
\hline Martinez WT + PT & 84.71 & 59.73 & 72.22 \\
\hline $\begin{array}{l}\text { Martinez WT + } \\
\text { templates }\end{array}$ & 88.50 & - & - \\
\hline MMF + Elgendi & 79.97 & 51.88 & 65.92 \\
\hline
\end{tabular}

\section{Discussion and conclusions}

In this paper, we presented a novel approach for the detection of $\mathrm{P}$ and $\mathrm{T}$ peaks. The proposed methodology displays good F1 for the detection of both peaks.

In particular, on the QTDB our algorithm obtained considerably higher performance than those in the literature [7] for both P (95.87\% vs $89.05 \%$ for Lead 1 and $92.52 \%$ vs $86.92 \%$ for Lead 2$)$ and $\mathrm{T}$ wave $(92.52 \%$ vs $87.49 \%$ for Lead 1 and $88.70 \%$ vs $88.50 \%$ for Lead 2) detection. On the MIT-BIH database our results were 
better than that of the literature for $\mathrm{P}$ wave detection (84.65\% vs $83.37 \%$ for Lead 1 and $78.32 \%$ vs $78.09 \%$ for Lead 2) and almost comparable to those reported in the literature for $\mathrm{T}$ wave detection.

Notably, the detection of Tpeak, which gave lower performance compared with Ppeak, is somehow complicated by the fact that different shapes of the wave exist in the databases, as shown in Figure 1. Moreover, Elgendi et al. [5] often did not annotate the inverted $\mathrm{T}$ waves, which is visible when inspecting both leads.

It is important to underline that in [7] the best performances were not obtained with the same algorithm. The algorithms with the best performances were: i) the one by Martinez et al. [13] based on wavelet transformation (WT) approach; ii) the combination of this algorithm with Martinez's PT algorithm based on phasor transform (PT) [14]; iii) the WT algorithm with window parameter modification based on waveform templates, as introduced in [6]; iv) the algorithm by Elgendi et al. based on moving averages [15] with mathematical morphological filtering (MMF) for noise reduction and baseline correction [16].

Although the three algorithms based on Martinez WT approach obtained high performance on QTDB, the performance on MIT-BITH database were quite poor, especially for $\mathrm{T}$ wave. On the contrary, while the algorithm by Elgendi was the one who achieved the best performance for T wave detection on MIT-BITH database in Lead 1, its performance was very low in Lead 2 and quite low for QTDB.

Observing the mean value of the performance of the different algorithms for the two leads of the two databases (Table 3 and Table 4), it is evident that ours are the ones with the best mean value which is far higher than the others algorithm. Thus, the strength of our algorithms for peak detection is their high stability and generalizability so that the performance remains high while changing the dataset.

The presented algorithms are simple in their approach and so they are applicable also in the contests in which low computational cost is required such as real-time applications. These findings suggest the high potential of the proposed approach for P and T wave detection in ECG.

\section{Acknowledgments}

This work was funded from Cardioline S.p.A. and from the Italian MIUR through the PRIN project "Preclinical Tool for Advanced Translational Research with Ultrashort and Ultraintense X-ray Pulse” (prot. 20154F48P9).

\section{References}

[1] E. Braunwald, D. Zipes, P. Libby, R. Bonow, "Braunwald's Heart Disease: A Textbook of Cardiovascular Medicine," 10th ed.; Saunders: Philadelphia, PA, USA, 2004.

[2] G. Carrault, M. Cordier, R. Quiniou, F. Wang F, “Temporal Abstraction and Inductive Logic Programming for
Arrhythmia Recognition from Electrocardiogram," Artif. Intell. Med., vol. 28, pp. 231-63, 2003.

[3] H. Purerfellner, E. Pokushalov, S. Sarkar, J. Koehler, R. Zhou, L. Urban, G. Hindricks, "P-wave Evidence as a Method for Improving Algorithm to Detect Atrial Fibrillation in Insertable Cardiac Monitors," Hear Rhythm, vol. 11, pp. 1575-1583, 2014.

[4] L. Billeci, M. Costi, D. Lombardi, F. Chiarugi, M. Varanini, "Automatic Detection of Atrial Fibrillation and Other Arrhythmias in ECG Recordings Acquired by a Smartphone Device," Electronics, vol. 7, 199, 2018.

[5] M. Elgendi, B. Eskofier, D. Abbott, "Fast T Wave Detection Calibrated by Clinical Knowledge with Annotation of $\mathrm{P}$ and T Waves," Sensors, vol. 15, pp. 17693-17714, 2015.

[6] N. Ku. Dewangan1, M. K. Kowar, "A Review on ECG Signal De-noising, QRS Complex, $\mathrm{P}$ and $\mathrm{T}$ Wave Detection Techniques," IJIREEICE, vol. 3, pp. 10-14, 2015.K.

[7] K. Friganovic, D. Kukolja, A. Jovic, M. Cifrek and G. Krstacic, "Optimizing the Detection of Characteristic Waves in ECG Based on Processing Methods Combinations," IEEE Access, vol. 6, pp. 50609-50626, 2018.

[8] G. B. Moody and R. G. Mark, "The Impact of the MIT-BIH Arrhythmia Database," IEEE Eng. Med. Biol. Mag., vol. 20, pp. 45-50, 2001.

[9] P. Laguna, R. G. Mark, A. Goldberg, and G. B. Moody, “A Database for Evaluation of Algorithms for Measurement of QT and other Waveform Intervals in the ECG," in: Proc. Comput. Cardiol. 1997, vol. 24, Boston, MA, USA, pp. 673676, 1997.

[10] I. Christov, and I. Simova, "Fully Automated Method for QT Interval Measurement in ECG," in: Proc. Comput. Cardiol. 2006, vol. 33, pp. 321-324, 2006.

[11] H. C. Bazett, "An Analysis of the Time-Relations of Electrocardiograms," Heart, vol. 2, pp. 7353-7370, 1920.

[12] AAMI-ECAR, "Recommended Practice for Testing and Reporting Performance Results of Ventricular Arrhythmia Detection Algorithms," Assoc. Adv. Med. Instrum., 1987.

[13] J. P. Martinez, R. Almeida, S. Olmos, A. P. Rocha, and P. Laguna, "A Wavelet-Based ECG Delineator Evaluation on Standard Databases," IEEE Trans. Biomed. Eng., vol. 51, pp. 570-581, 2004.

[14] A. Martínez, R. Alcaraz, and J. J. Rieta, "A New Method for Automatic Delineation of ECG Fiducial Points Based on the Phasor Transform," in: Proc. 2010 Annu. Int. Conf. IEEE Eng. Med. Biol. Soc. EMBC'10, Buenos Aires, Argentina, 2010, pp. 4586-4589.

[15] M. Elgendi, M. Meo, and D. Abbott, "A Proof-of-Concept Study: Simple and Effective Detection of $\mathrm{P}$ and $\mathrm{T}$ Waves in Arrhythmic ECG Signals," Bioengineering, vol. 3, p. 26, 2016.

[16] Y. Sun, K. L. Chan, and S. M. Krishnan, "ECG Signal Conditioning by Morphological Filtering," Comput. Biol. Med., vol. 32, pp. 465-479, 2002.

Address for correspondence:

Lucia Billeci

Institute of Clinical Physiology, National Research Council of Italy (IFC-CNR),

via Moruzzi 1, 56124, Pisa, Italy

lucia.billeci@ifc.cnr.it 